\title{
Care protocol for acute traumatic tissue injuries in prehospital mobile service
}

\author{
Rodrigo Pereira Bettega * (1), Renato Santos de Oliveira Filho' ${ }^{1}$, Alexandre Soares Lavorato ${ }^{2}$ (1), \\ Christiane Steponavicius Sobral ${ }^{1,3}$ (1), Lydia Masako Ferreira ${ }^{3}$ (1)
}

\section{SUMMARY}

OBJECTIVE: The aim of this study is to elaborate a protocol for the care of acute traumatic tissue injuries in the prehospital mobile service. METHODS: An extensive search of anteriority that did not reveal the existence of such protocol in other services across the globe was carried out. Subsequently, the literature review was executed on Medline and Lilacs. Related and referenced scientific literature served as the basis for the elaboration and creation of the protocol and its flowchart. The complete protocol was then measured for expert judges with the Delphi methodology. A questionnaire (using the Likert scale) and the initial version protocol were sent by electronic means to 13 medical and nurse coordinators of all SAMU from Paraná. RESULTS: Seven experts returned completed questionnaires in the first round and five in the second round. Average required global content validation index was obtained for the validation requirements of the protocol and its flowchart.

CONCLUSIONS: A protocol for the care of acute traumatic tissue injuries in the prehospital mobile service was developed and authenticated with the viability of routine professional use by the health professional.

KEYWORDS: Emergency medical services. Wounds and injuries. Clinical protocols.

\section{INTRODUCTION}

The advent of the industrial era, advanced technology, increased vehicle speed, socioeconomic conditions, poverty, and human nature itself are the factors that have contributed to the progressive increase in different types of trauma ${ }^{1}$. This rise is a public health problem due to the high associated mortality, morbidity, costs, years of potential life lost, and impact on the individual, their family, and society ${ }^{2}$.

Appropriate first care to trauma victims, right at the event, with the proper approach to injuries, directly affects morbidity and mortality of patients. Moreover, the costs and quality improvement of the initial approach are related to the level of prehospital care (PHC). All these factors together have a major impact on the progress of severe traumatic injury.
Updated intra-hospital protocols that standardize and organize care have previously been published, and they should be used as routine by all health professionals. However, there is no such protocol for PHC in Brazil or any other country to be used outside of hospitals.

\section{METHODS}

This is a primary, descriptive, single-center study. There was no sponsorship from any public or private entity for the development of this study. Possible conflicts of interest may have arisen from the primary author's employment relationship with the Londrina Northern MacroRegional SAMU.

\footnotetext{
'Universidade Federal de São Paulo, Departamento de Ciência, Tecnologia e Gestão Aplicadas à Regeneração Tecidual - São Paulo (SP), Brazil.

¿Universidade Nove de Julho - São Paulo (SP), Brazil.

${ }^{3}$ Universidade Federal de São Paulo, Departamento de Cirurgia Plástica - São Paulo (SP), Brazil.

*Corresponding author: rpbettega@gmail.com

Conflicts of interest: the authors declare there is no conflicts of interest. Funding: none.

Received on April 30, 2021. Accepted on May 30, 2021.
} 
This project was approved by CEP/UNIFESP under No. CAAE 05053218,1,0000,5505 and was conducted according to the ethical standards of the Declaration of Helsinki and its amendments. All participants provided informed consent to take part in this study, and their anonymity was ensured.

This study was divided into the following three stages:

1. Prior article search, bibliographic survey, content selection, and information treatment;

2. Protocol development, drafting, and creation of the flowchart with layout resources;

3. Protocol validation by urgency and emergency professionals working in PHC (SAMU).

A search of previous studies was first conducted in non-scientific research platforms and websites (i.e., Google Scholar and Yahoo) to reinforce the need for the proposed protocol. Subsequently, a literature search was performed in the Medline, Scielo, and Lilacs databases using the descriptors' emergency medical services, SAMU, protocols, injuries, and wounds, over the past 10 years.

The original content for the protocol was obtained from the fractional scientific literature references provided at the end of the document. All the information found on injuries and traumatic wounds was selected, compiled, summarized, critically analyzed, and used in the final formatting.

The protocol was written in technical language for the use of health professionals, specifically physicians and nurses.

The AGREE II instrument (AGREE Next Steps Consortium, 2009) was used as a tool to model the construction of the protocol body text. Its initial objectives were as follows:

1. To analyze the quality of clinical guidelines;

2. To provide a methodological strategy for the development of clinical guidelines;

3. To report what and how information should be reported in clinical guidelines;

4. To confirm the strength of evidence of the sources used in the development of clinical guidelines.

The evaluation of the protocol content and layout was performed by 13 expert judges using the Delphi method.

The specialists received an invitation letter by e-mail explaining the objective of the study, along with the informed consent form (ICF) and a questionnaire (via Google forms) developed for protocol evaluation.

The questionnaire items presented five responses as follows:

1. Inadequate;

2. Partially adequate;

3. Adequate;
4. Very adequate; and

5. N/A = not applicable.

The content validity index (CVI) was used to measure the percentage of judges who agreed with several aspects of the manual. Thus, the CVI was estimated considering the number of adequate or very adequate responses ( 3 and 4 ) for each item, divided by the total number of answers.

The questionnaire evaluated three main topics as follows:

1. Objective (6 items), evaluating the importance of the subject;

2. Structure and presentation (11 items), analyzing the overall presentation, general organization, structure, presentation strategy, coherence, and formatting; and

3. Relevance (4 items).

The selection of experts was conducted by non-probability sampling from a sample the researcher had access to, considering that the sample was properly representative ${ }^{3}$.

The inclusion criteria for experts were active positions as medical and nursing coordinators of the SAMU in the state of Paraná and agreement to participate by signing the ICF. There were no exclusion criteria, except for not meeting the inclusion criteria. In case of participant dropout, another member was chosen from the invited group.

\section{RESULTS}

The final result of this study was a clinical protocol validated by health professionals who work directly in PHC. This study was analyzed by the medical and nursing coordinators of the SAMU in the state of Paraná who develop activities related to research (Delphi method).

To start the protocol validation, an invitation, a questionnaire, a copy of the protocol, and the ICF were sent via e-mail to each selected professional. Of the 13 experts invited, 7 returned the completed questionnaire in the first round of Delphi and 5 in the second round, performed 2 months after the first round.

A limit of three rounds was previously stipulated by the researcher for the submissions and corrections of the material being elaborated. The consensus was already reached in the first round, and the second round was repeated to confirm the methodology. This rapid positive result is due to the fact that most of the protocol items were based on the PHC professionals' daily practice and common sense. The final overall CVI of the protocol was 0.95 in the first round and 0.98 in the second round, resulting in a final mean CVI of 0.96 . 
At the end of the document, an algorithm of the care procedures to be performed by the health professional was presented in a simplified and easy-to-follow flowchart. The final protocol has a total of seven pages.

Annex 1 shows the complete protocol for viewing and use. The final flowchart is focused, which was carefully developed with every detail. Simple colors were used for didactic purposes and for better visualization of the sources of information. The objective was to gradually draw attention to specific parts of the flowchart text. Its design is unique and simple, with an artistic, aesthetic, and creative look, giving the document its own identity.

\section{DISCUSSION}

The motivation for this study originates from the "pain" related to the difficulty of prehospital mobile care. These difficulties start during the assistance process and continue during the patients' arrival at their destination hospitals for therapeutic follow-up. This lack of flow within the SAMU service in the state of Paraná highlights the need for a care protocol to allow standardization, formatting, and prioritization when assisting victims with acute traumatic tissue injuries.

An article by Jones et al. ${ }^{4}$, reporting the lack of protocols for PHC in the United Kingdom, corroborates this study. The teams reported in that article had disorganized and non-standardized behaviors, with no guides or protocols. In a new study, Jones et al. ${ }^{5}$ showed this same reality in the emergency departments of hospitals in the same country. This knowledge further motivated the search for models and flowcharts to assist the work of health professionals.

Dantas et $\mathrm{al}^{6}{ }^{6}$ showed the reality of a town in the state of Bahia, Brazil. The discussion of this local epidemiological profile can be generalized to the entire Brazilian reality. The statistical data found were similar to those in all other studies in the past 10 years in Brazil, confirming the need for improving assistance to traffic accidents in PHC.

The AGREE II (2009) instrument was chosen as the basis for the development of the protocol due to its free access, ease of application, and wide use in health services. The Delphi method, with Likert scale scores, was chosen for the validation of the final product. This methodology was selected for its ease of use and quick applicability in the field throughout validation. The Delphi method, used for validation, can be used to obtain opinions and criteria from a group of specialists on a given topic. It uses questionnaires, and in each phase, information from previous phases is used in the search for consensus among experts?
Cunha et al. ${ }^{8}$ wrote the scientific article that methodologically motivated and guided the preparation of this study, with a similar structure and methodology. The studies by Rezende et al. ${ }^{9}$ and Brown et al. ${ }^{10}$ also served as a basis for the development of a flowchart with potential use throughout the Brazilian SAMU, respecting local and general needs. A flowchart associated with an organized protocol has considerable applicability potential.

After construction of the protocol with the final version of the flowchart and complete validation by the expert judges, the next step was its dissemination among potential users. The protocol was submitted to the Brazilian ISBN Agency and received its numerical cataloging and the barcode for future references. It was also presented to other services of prehospital mobile care of SAMU in the state of Paraná and was very well accepted. Several requests for the use of the full protocol by other services have already been sent via e-mail.

A subsequent potential analysis of the implementation of the document in the various micro-regional realities of Paraná in its regional SAMUs could be analyzed in a future study. Furthermore, its use could be applied to many other services in Brazil. Each micro or macro region of the country has a different reality, which could be a limitation. However, in the future, this protocol may be adapted according to the region.

The statistical data generated from the use of this protocol in clinical practice, with tabulation and critical analysis, indicate the availability of further information on this theme. In the researcher's prognostic view, this is a document that will favorably impact the economy and the health of those who suffer traumatic tissue injuries.

\section{AUTHORS' CONTRIBUTIONS}

RPB: Conceptualization, Data curation, Formal analysis, Funding acquisition, Investigation, Methodology, Project administration, Resources, Software, Supervision, Validation, Visualization, Writing - original draft, Writing - review \& editing. RSOF: Conceptualization, Data curation, Formal analysis, Funding acquisition, Investigation, Methodology, Project administration, Resources, Software, Supervision, Validation, Visualization, Writing - original draft, Writing - review \& editing. ASL: Conceptualization, Data curation, methodology, project management, Resources, Software, Supervision, Validation, Visualization, Writing - original draft, Writing - review \& editing. CSS: Conceptualization, Methodology, Supervision, Validation, Visualization, Writing - original draft, Writing - review \& editing. LMF: Conceptualization, Methodology, Supervision, Validation, Writing - original draft, Writing - review \& editing. 


\section{REFERENCES}

1. Cardona D, Peláez E, Aidar T, Ribotta B, Alvarez MF. Mortalidad por causas externas em tres ciudades latinoamericanas: Córdoba (Argentina), Campinas (Brazil) y Medellín (Colombia), 1980-2005. R Bras Est Pop. 2008;25(2):335-52. https://doi. org/10.1590/S0102-30982008000200009

2. Caixeta CR, Minamisava R, Oliveira LMAC, Brasil VV. Traffic injuries among youth in Goiânia, Goiás State. Cienc Saude Colet. 2010;15(4):2075-84. https://doi.org/10.1590/s141381232010000400021

3. Polit DF, Beck $C T$, Hungler BP. Análise quantitativa. In: Polit DF, Beck CT, Hungler BP, eds. Fundamentos de pesquisa em enfermagem: métodos, avaliação e utilização. $5^{\text {a }}$ ed. Porto Alegre: Artmed; 2004. p. 167-98.

4. Jones AP, Allison $\mathrm{K}$, Wright $\mathrm{H}$, Porter $\mathrm{K}$. Use of prehospital dressings in soft tissue trauma: is there any conformity or plan? Emerg Med J. 2009;26(7):532-4. https://doi.org/10.1136/ emj.2008.058255

5. Jones AP, Barnard AR, Allison K, Wright H. Review of emergency department wound management in soft tissue trauma - is there a plan? J Wound Care. 2012;2(9):431-4, 436, 438. https://doi.org/10.12968/jowc.2012.21.9.431
6. Dantas GSV, Rios MA, Silva JK, Pereira DC, Fonseca EOS. Perfil dos acidentes motociclísticos atendidos pelo Serviço de Atendimento Móvel de Urgência nos anos de 2014 e 2015 em município baiano. Rev Pesqui Cuid Fundam. 2019;11(4):984-91.

7. Faro ACM. Técnica de Delphi na validação das intervenções de enfermagem. Rev Esc Enferm USP. 1997;31(2):259-73.

8. Cunha DR, Salomé GM, Massahud Junior MR, Mendes $B$, Ferreira LM. Development and validation of an algorithm for laser application in wound treatment. Rev Latino-Am Enfermagem. 2017;25:e2955. http://doi.org/10.1590/15188345.1998.2955.

9. Rezende RBM, Macedo JLS, Rosa SC, Galli FS. Epidemiological profile and treatment of substance losses by trauma to the lower limbs. Rev Col Bras Cir. 2017;44(5):444-51. https://doi. org/10.1590/0100-69912017005004

10. Brown KM, Hirshon JM, Alcorta R, Weik TS, Lawner B, Ho S, et al. The implementation and evaluation of an evidence-based statewide prehospital pain management protocol developed using the national prehospital evidence-based guideline model process for emergency medical services. Prehosp Emerg Care. 2014;18 Suppl 1:45-51. https://doi.org/10.3109/10903127. 2013.831510

Annex 1. Care protocol for acute traumatic tissue injuries in prehospital mobile service.

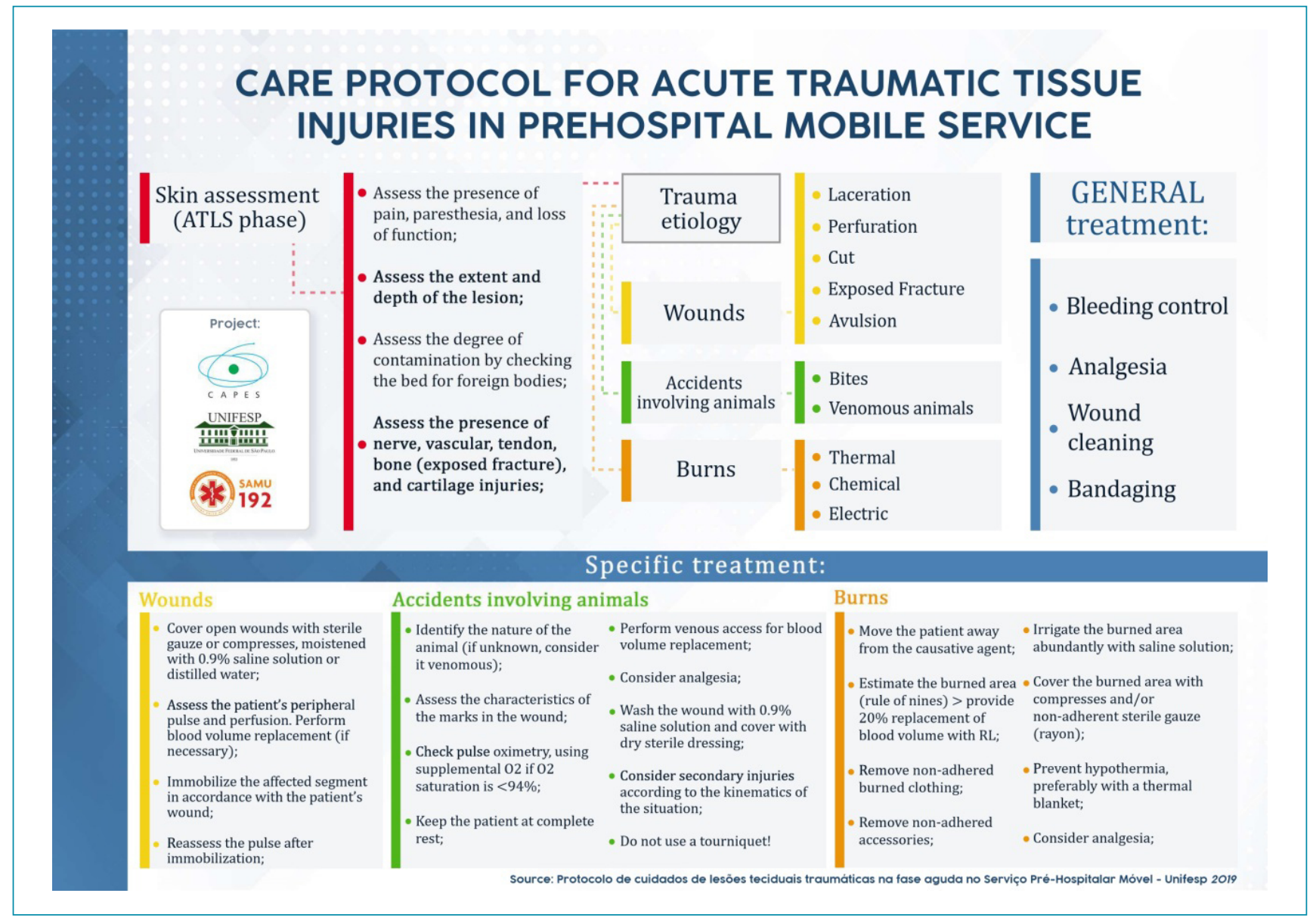

